\title{
Comparison of VM Live Migration Process Impacts on QoS of Web Server Hosted in Cloud
}

\author{
Matheus Torquato \\ Federal Institute of Alagoas, Campus Arapiraca \\ Arapiraca, AL - Brazil
}

\begin{abstract}
Virtual Machine (VM) Live Migration improves system manageability through VMs rearrangement in their physical hosts. System managers can redistribute VMs to tackle different challenges as: VMs consolidation for energy consumption reduction, load balancing and software rejuvenation. VM live migration consists in successive transfers of memory pages from one host to another. Thus, system capacity planning may consider VM live migration overhead. This paper presents impacts of VM live migration operations on system performance of different RAM-sized VMs (512MB, 1GB and 2GB). These experiments aims to observe how RAM size changes can affect responsiveness and reliability of a Web Server hosted on a single VM. The experiment has external client which sends web requests in a constant rate through Autobench (httperf) benchmark tool. This benchmark tool provides results of Web Server errors and response time during VM live migration process. Experiments results also consider network throughput during VM live migration. The results presents that network overhead due to VM live migration causes major system performance degradation in VMs with more amount of RAM. Therefore, this paper results may be useful for managers to consider VM live migration impacts in capacity planning.
\end{abstract}

\section{General Terms}

Cloud Computing, Capacity Planning

\section{Keywords}

VM Live Migration, Cloud Computing, Performance, Web Server

\section{INTRODUCTION}

Many companies around the world relies in Cloud Computing to run and host their services. One of cores of Cloud Computing is the virtualization technology [9]. Virtualization enables fast service deployment and improves system manageability. The virtualization technology consists in a technique to decouple hardware resources from a Operating System [29]. Among features of virtualization technology, Virtual Machine (VM) live migration appears as important feature to improve system manageability [7].

VM live migration process consists in move a VM from one Physical Machine (PM) to another. Thus, it is possible to transfer services to other host before perform system maintenance; or load balacing; or software rejuvenation [20]. Algorithms of VM live migration contains a process of transfer VM memory pages between physical hosts. This process consumes computational resources and led system to overhead. In final phases, VM live migration process interrupt service delivery by switching income requests to PM target of migration. Overhead and downtime can not be neglected in system capacity planning [13]. There are different techniques to improve quality of service during VM live migration [21]. Section 2 contains further details about VM live migration.

Generally, companies move their applications to Cloud Computing environment to improve quality of service (QoS). QoS of a service consist in perceived delay of majority of packets stays below a certain threshold [3]. In particular, with explosive growth of Web, the performance of Web servers is key factor for success of companies [11]. Among several types of Web Server, we can highlight Apache Web server due to its several side projects attached and popularity [1].

In this paper, we present a study of VM live migration impacts on QoS of Web Server hosted in private Clouds. The testbed consists in a OpenNebula Cloud with three Execution Nodes, one FrontEnd and one Web Server VM (more details in Section 3). First step of experimentation aims to stress a single VM with a increasing workload of requests. The results provides a tolerable workload to Web Server hosted in VM. The main experiment consists in compare different RAM-sized VMs (512MB, 1GB and 2GB) with same workload during VM live migration. Section 4 shows details about methodology adopted in our experiments. Experiments results reveals that VMs with more RAM harms network throughput which leads to QoS degradation. Results also shows that response Time and errors of Web Server hosted in VMs with more RAM has worse results than others. The Section 5 has experiments results.

In face of other previous published works, this papers presents a comparison between different RAM-sized VMs. Results can be useful for system manager in their capacity planning projects. Section 6 presents related works. Future research directions aims to test more performance metrics of Web Server and also test a Software Defined Network (SDN) for further experimentation. The conclusions and future works are in Section 7 


\section{VM LIVE MIGRATION}

On VDs, VMs host main services (e.g. Web Server, Mail Server) which runs on PMs [5]. Thus, VMs depends of PMs to deliver correct service to its users. The Figure 1 presents a simplified schema of a traditional VDC.

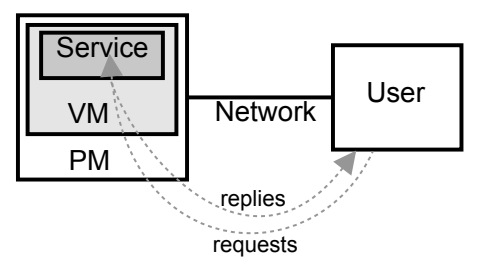

Fig. 1. A traditional VDC schema

Perform maintenance on PMs which runs VMs causes service downtime. On VDCs environments is possible to mitigate this problem by migrating VMs and its services to another PM [7]. Besides, there are other reasons to migrate a VM, as load balancing [28] and support for software rejuvenation [27, 19].

There are different techniques to implement VM live migration on VDC environment. The technique used in this paper is the precopy technique. Previous studies use precopy approach as default technique [7 23]. And this technique is also default for well-known hypervisors as KVM and Xen [14, 4]. Precopy approach has six main phases [7]. Figure 2 summarizes VM live migration precopy approach.

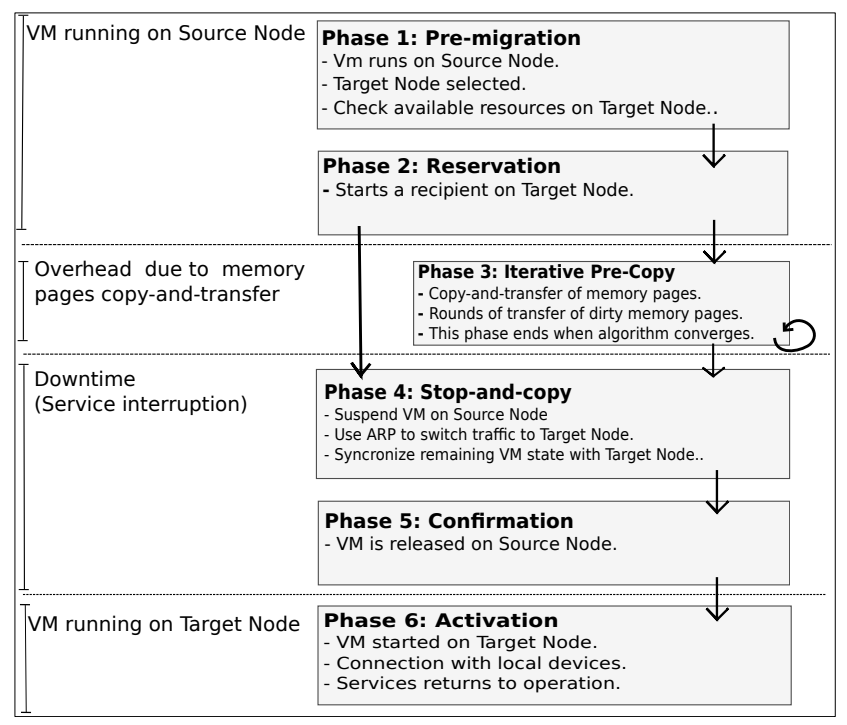

Fig. 2. Precopy VM Live Migration Approach (adopted from [7])

Among other VM live migration techniques it is possible to highlight: (i) Live VM Migration with Adaptive Memory Compression - compress memory pages to achieve fast and stable machine migration [12]; (ii) Post-copy - this technique consists in transfer processor state in first phase of migration, this allows target host to continue system processing in early phases of migration process [10]; (iii) Full trace and replay - source machine transfer system logs to target machine which replays it [17]. Previous studies [16] 24, 25] present surveys of different VM live migration techniques.

\section{SYSTEM ARCHITECTURE}

The testbed used for experiments consists in a private Cloud built upon OpenNebula 3.4 Manager. This environment has three PMs acting as Execution Nodes (Node01, Node02, Node03) and one PM as Front End (FrontEnd). A single VM (VM-WS) has Apache2 Web Server with a simple HTML page called teste.html. An external machine (Workload Generator) sends workload to VM-WS using httperf [22] as benchmark tool. The main objective is to perceive possible performance impacts during VM live migration process. A private network switch connects all components. Figure 3 presents testbed architecture. Table 1 shows hardware and software configuration for architecture components.

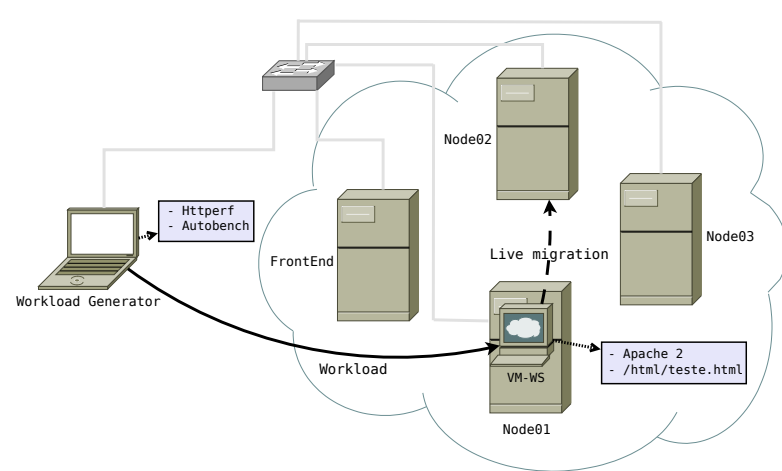

Fig. 3. Testbed Architecture

Table 1. Components configuration

\begin{tabular}{|l|l|l|}
\hline Component & Hardware & Software \\
\hline $\begin{array}{l}\text { FrontEnd, } \\
\text { Node01, Node02, }\end{array}$ & $\begin{array}{l}\text { AMD Phenom 9600B } \\
\text { Quad-Core Processor, } \\
\text { 4GB RAM, 250GB HDD }\end{array}$ & $\begin{array}{l}\text { Ubuntu Server, } \\
\text { KVM Hypervisor } \\
\text { and OpenNebula } \\
\text { Manager }\end{array}$ \\
\hline $\begin{array}{l}\text { Workload } \\
\text { Generator }\end{array}$ & $\begin{array}{l}\text { Intel Core i5 2410M } \\
2.30 \mathrm{GHz} \text { Processor, 4GB } \\
\text { RAM, 750GB HDD }\end{array}$ & $\begin{array}{l}\text { Ubuntu Desktop, } \\
\text { httperf, autobench }\end{array}$ \\
\hline VM-WS & $\begin{array}{l}\text { Single-core 1GHz Proces- } \\
\text { sor }\end{array}$ & $\begin{array}{l}\text { Ubuntu Server, } \\
\text { Apache2 Web } \\
\text { Server }\end{array}$ \\
\hline
\end{tabular}

\section{EXPERIMENT METHODOLOGY}

Experiment methodology has two main steps: (i) Preliminary experiment - Focused on Workload Selection and (ii) Main experiment - Focused on Web Server performance comparison among different VM configurations.

The preliminary experiment is a stress test Web Server hosted in VM-WS. Experiments on this phase aims to reveal VM-WS capacity to 
handle different requests rates. This is a simpler approach to conduct Web Server capacity planning. The paper [2] presents extensive view of Web Server capacity planning.

The experiments of both steps use httperf benchmark tool [22] with Autobench wrapper ${ }^{1}$ For preliminary experiment, $h$ ttperf tool was set to send web requests with increasing rate. The main objective is to perceive Web Server performance degradation when it is exposed to an increasing web request rate. Therefore, it is possible to proper request rate for Web Server configuration. The Web Server used was on a VM with 512MB of RAM.

Second phase of experiment (main experiment) consists in submit workload selected on previous phase to VMs with 512MB, 1GB and $2 \mathrm{~GB}$ of RAM. The test for each VM ran separately. This phase aims to observe and compare impacts of VM live migration process in performance of Web Server hosted on VM. The observation contains three main metrics:

(1) Response Time under Live Migration - The main metric of Web Server performance is response time. Results for this metric presents possible performance impacts during VM live migration for different RAM-sized VMs. Thus, it is possible to compare them.

(2) Amount of Errors observed under Live Migration - Other important metric is the amount of errors observed. Amount of errors produce lack of confidence in Web Server purpose. Httperf tool collects errors of connection timeout and socket timeout.

(3) Network Throughput under Live Migration - As VM live migration lies in memory pages network transfer, it is important to observe possible impacts on network throughput. Web Server requests and replies throughput are also transfer on network. If network is overloaded by VM live Migration transfer, quality of service of Web Server will be degraded.

The methodology of main experiment consists in a constant workload of 3000 requests per second to Web Server hosted in VM-WS. Each observation lasted 10 minutes. Results presents mean values for each metric observed in 30 successive rounds of VM live migration.

\section{RESULTS AND ANALYSIS}

\subsection{Preliminary experiment}

First phase of experiment is to send web requests on a increasing rate to a Web server hosted on a VM with 512MB RAM. Figure 4 presents experiment results. This preliminary experiment reveals that a Web Server performance becomes degraded after 5000 requests per second rate. Observing results is possible to notice that Web Server is capable to respond to 3000 requests/second with acceptable response time and near-zero errors. It is worth highlight that Web Server stressed carry a simple HTML page which has only text.

This experiment provides correct input configuration for benchmarking tool on main experiment.

\footnotetext{
${ }^{1}$ http://www.xenoclast.org/autobench/
}

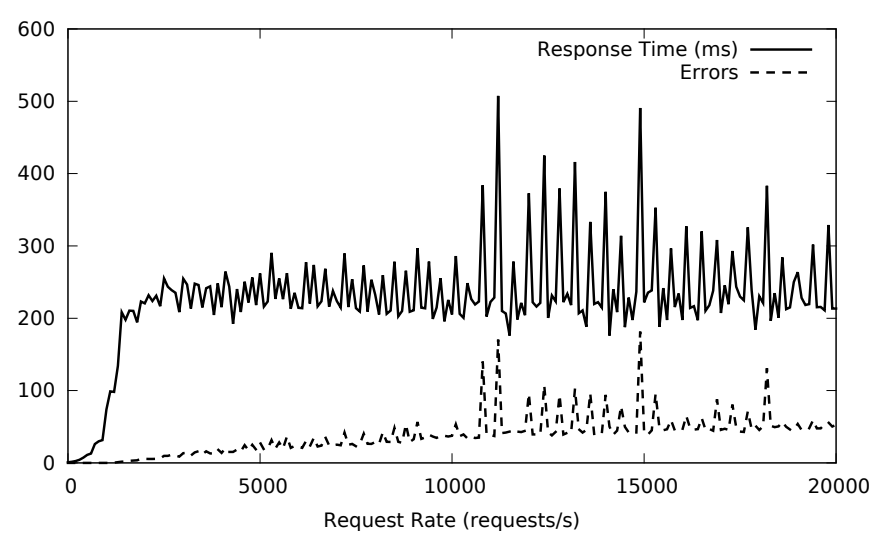

Fig. 4. Stress tests results

\subsection{Main experiment}

\section{Response Time.}

VM live migration operation requires computational resources (processing, network). This resources consumption may affect other services hosted on same VM. On a Web Server, main metric of QoS is response time. To perceive possible impacts on Web Server QoS, Figure 5 presents results of Response Time under Live Migration for 512MB, $1 \mathrm{~GB}$ and $2 \mathrm{~GB}$ RAM VMs.

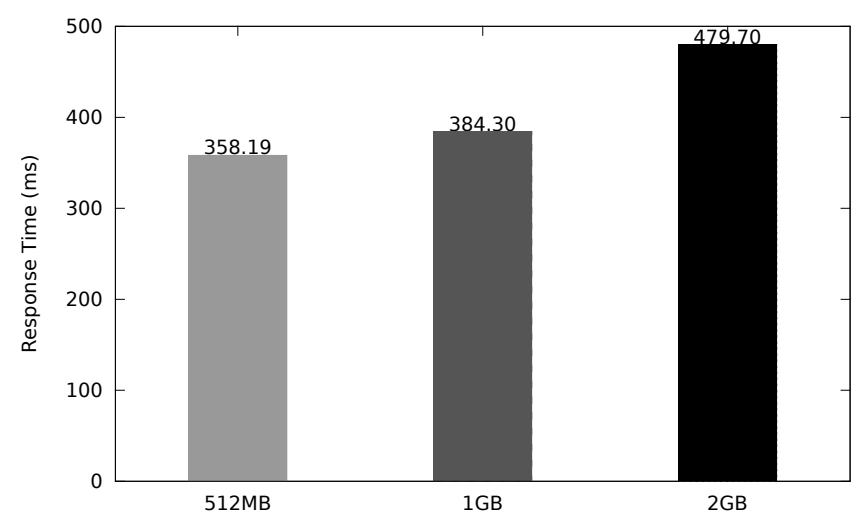

Fig. 5. Web Server Response Time (ms) under Live Migration

Response Time in VMs with more RAM are higher. Usually, systems with more resources have better performance. In this case, VM live migration produces a significant overhead in VMs with more RAM. Therefore, in this experiment, VM live migration brings QoS drawbacks in VMs with more RAM. VM live migration algorithm consists in transfer memory pages from one host to another, so VM with more RAM takes more time to finish the job. VM memory page transfer flow may cause overload to network.

Network Throughput. VM live migration relies on network communication to transfer memory pages of VM from one host to another. But, in some cases, network also carries other flows. In this 
experiment, network has to transfer traffic from Web Server requests and replies; and VM live migration memory pages. Figure 6 presents an overview of network communication during main experiment.

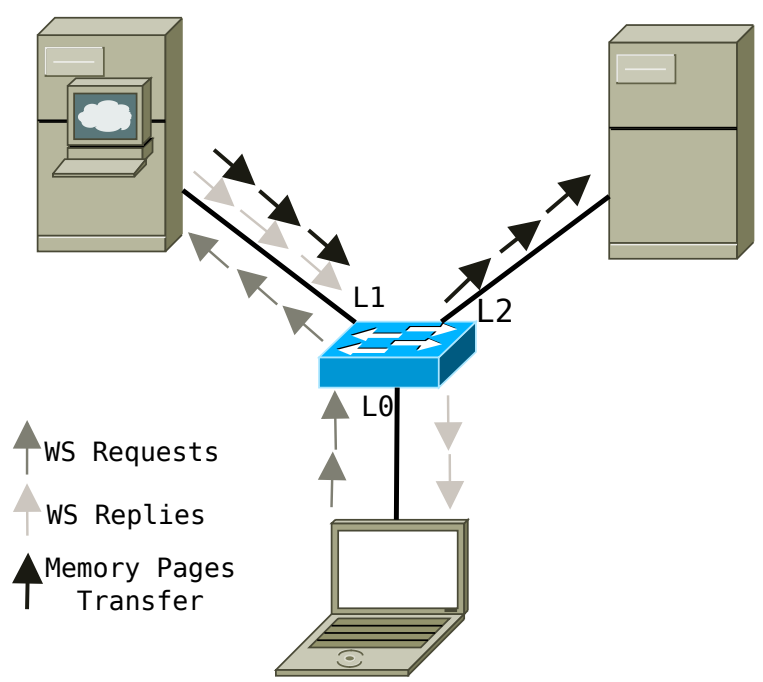

Fig. 6. Network traffic on first phases of Live Migration

The overload in link L1 may cause network throughput degradation. This effect can also affect quality of service. The Figure 7 presents results of network throughput for each VM tested.

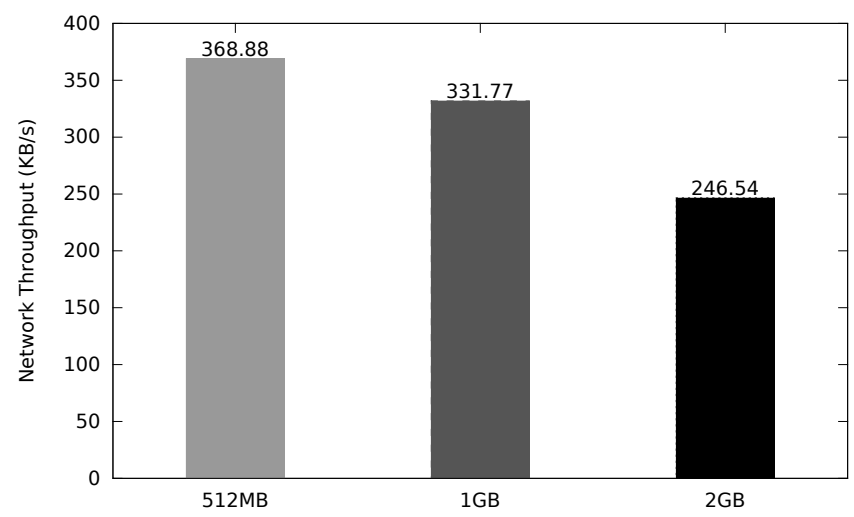

Fig. 7. Network Throughput under Live Migration

It is possible to perceive that VM with $2 \mathrm{~GB}$ of RAM has less network throughput than others. VM live migration of VMs with more RAM produces more QoS degradation. As bigger-RAM VM has more memory pages to transfer, VM live migration takes more time to converge. VM live migration flow fills network with other flows for longer time (in VMs with more RAM). Therefore, this phenomenon harms network throughput for Web Server requests and replies.
Errors.

Errors reflect system quality of service. Figure 8 presents experiment results for errors. It is possible to notice that errors are higher in VMs with more RAM. Because of network degradation (previous metric) system face more errors due to connection timeout and socket timeout.

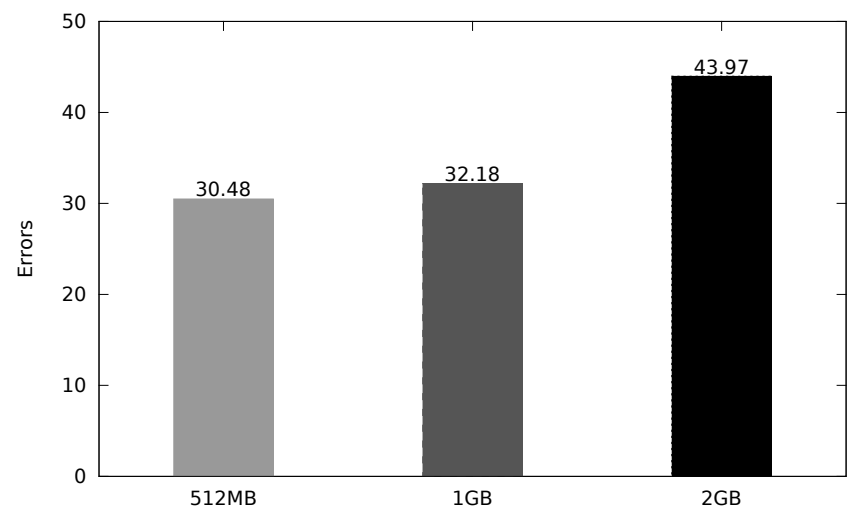

Fig. 8. Web Server Errors under Live Migration

\section{RELATED WORK}

Previous published works shows that VM live migration operation may cause different impacts on system performance and QoS. The paper [26] presents a comprehensive survey of cost of VM live migration. One of issues of VM live migration costs is performance loss of migrated VM. Kuno et al [15] presents a study of VM live migration process performance. In their study, they investigate VM live migration impacts on processing and throughput of CPU and performance in disc I/O intensive workloads. The work [6] compares results of VM live migration with fixed and variable network bandwidth. The paper [8] proposes a traffic-sensitive live VM migration technique which aims to reduce the contention of migration traffic with the VM application traffic. Zhao et al [30] provides a model to characterize VM migration process and predict its performance.

The work presented in [28] shows impacts of VM live migration operations by observing total downtime and response time during VM live migration. This work provides useful insights for methodology used in our methodology. The paper [18] also presents useful thoughts for selection of significant metrics for our study. This paper presents results of an experiment using Olio benchmark to stress a system on a VM. The results shows impacts caused by VM live migration operation on system overall performance.

Most of collected papers has different approaches to study VM live migration impacts on system performance and quality of service. Unlike previous published works, our paper aims in compare VMs with different amounts of RAM carrying same service and exposed to same workload. Our purpose is to perceive possible impacts in this environment due to VM live migration.

\section{CONCLUSION AND FUTURE WORKS}


This paper presented a study about impacts of VM live migration on quality of service of Web Servers hosted in VMs on a private cloud. The results shows that VM with more RAM has less QoS than minor VMs. VMs with more RAM has worstresults. Therefore, system capacity planning may consider these impacts. For example, system manager may choose two distributed VMs of 512MB RAM instead one of 1GB RAM to avoid large impacts due to VM live migration.

Next research efforts aims to deep studies about VM live migration operation in other metrics as: system overall downtime and internal resources consumption. Other lines of study aims to investigate VM live migration impacts under a software defined network (SDN).

\section{REFERENCES}

[1] The apache software foundation - http://www.apache.org/.

[2] Virgílio AF Almeida and Daniel A Menascé. Capacity planning an essential tool for managing web services. IT professional, 4(4):33-38, 2002.

[3] Matthew Andrews, Krishnan Kumaran, Kavita Ramanan, Alexander Stolyar, Phil Whiting, and Rajiv Vijayakumar. Providing quality of service over a shared wireless link. IEEE Communications magazine, 39(2):150-154, 2001.

[4] Paul Barham, Boris Dragovic, Keir Fraser, Steven Hand, Tim Harris, Alex Ho, Rolf Neugebauer, Ian Pratt, and Andrew Warfield. Xen and the art of virtualization. ACM SIGOPS Operating Systems Review, 37(5):164-177, 2003.

[5] M Faizul Bari, Raouf Boutaba, Rafael Esteves, Lisandro Z Granville, Maxim Podlesny, Md Golam Rabbani, Qi Zhang, and Mohamed Faten Zhani. Data center network virtualization: A survey. Communications Surveys \& Tutorials, IEEE, 15(2):909-928, 2013.

[6] David Breitgand, Gilad Kutiel, and Danny Raz. Cost-aware live migration of services in the cloud. In SYSTOR, 2010.

[7] Christopher Clark, Keir Fraser, Steven Hand, Jacob Gorm Hansen, Eric Jul, Christian Limpach, Ian Pratt, and Andrew Warfield. Live migration of virtual machines. In Proceedings of the 2nd conference on Symposium on Networked Systems Design \& Implementation - Volume 2, NSDI'05, pages 273286, Berkeley, CA, USA, 2005. USENIX Association.

[8] Umesh Deshpande and Kate Keahey. Traffic-sensitive live migration of virtual machines. In Cluster, Cloud and Grid Computing (CCGrid), 2015 15th IEEE/ACM International Symposium on, pages 51-60. IEEE, 2015.

[9] Chunye Gong, Jie Liu, Qiang Zhang, Haitao Chen, and Zhenghu Gong. The characteristics of cloud computing. 2010 39th International Conference on Parallel Processing Workshops, pages 275-279, 2010.

[10] Michael R. Hines, Umesh Deshpande, and Kartik Gopalan. Post-copy live migration of virtual machines. SIGOPS Oper. Syst. Rev., 43(3):14-26, July 2009.

[11] Yiming Hu, Ashwini Nanda, and Qing Yang. Measurement, analysis and performance improvement of the apache web server. In Performance, Computing and Communications Conference, 1999 IEEE International, pages 261-267. IEEE, 1999.

[12] Hai Jin, Li Deng, Song Wu, Xuanhua Shi, and Xiaodong Pan. Live virtual machine migration with adaptive, memory compression. In Cluster Computing and Workshops, 2009.
CLUSTER'09. IEEE International Conference on, pages 110. IEEE, 2009.

[13] Divya Kapil, Emmanuel S Pilli, and Ramesh C Joshi. Live virtual machine migration techniques: Survey and research challenges. In Advance Computing Conference (IACC), 2013 IEEE 3rd International, pages 963-969. IEEE, 2013.

[14] Avi Kivity, Yaniv Kamay, Dor Laor, Uri Lublin, and Anthony Liguori. kvm: the linux virtual machine monitor. In Proceedings of the Linux symposium, volume 1, pages 225-230, 2007.

[15] Y. Kuno, K. Nii, and S. Yamaguchi. A study on performance of processes in migrating virtual machines. In $A u$ tonomous Decentralized Systems (ISADS), 2011 10th International Symposium on, pages 567-572, March 2011.

[16] P.G.J. Leelipushpam and J. Sharmila. Live vm migration techniques in cloud environment - a survey. In Information Communication Technologies (ICT), 2013 IEEE Conference on, pages 408-413, April 2013.

[17] Haikun Liu, Hai Jin, Xiaofei Liao, Liting Hu, and Chen $\mathrm{Yu}$. Live migration of virtual machine based on full system trace and replay. In Proceedings of the 18th ACM international symposium on High performance distributed computing, HPDC '09, pages 101-110, New York, NY, USA, 2009. ACM.

[18] Deborah V Magalhães, José Marques Soares, and Danielo G Gomes. Análise do impacto de migração de máquinas virtuais em ambiente computacional virtualizado.

[19] M. Melo, J. Araujo, R. Matos, J. Menezes, and P. Maciel. Comparative analysis of migration-based rejuvenation schedules on cloud availability. In Systems, Man, and Cybernetics (SMC), 2013 IEEE International Conference on, pages 41104115, Oct 2013.

[20] M. Melo, P. Maciel, J. Araujo, R. Matos, and C. Araujo. Availability study on cloud computing environments: Live migration as a rejuvenation mechanism. In Dependable Systems and Networks (DSN), 2013 43rd Annual IEEE/IFIP International Conference on, pages 1-6, 2013.

[21] Anju Mohan and S Shine. Survey on live vm migration techniques. International Journal of Advanced Research in Computer Engineering and Technology, 2(1):155-157, 2013.

[22] David Mosberger and Tai Jin. Httperf - a tool for measuring web server performance. SIGMETRICS Perform. Eval. Rev., 26(3):31-37, December 1998.

[23] Michael Nelson, Beng-Hong Lim, Greg Hutchins, et al. Fast transparent migration for virtual machines. In USENIX Annual technical conference, general track, pages 391-394, 2005.

[24] Pradip D. Patel, Miren Karamta, M. D. Bhavsar, and M. B. Potdar. Live virtual machine migration techniques in cloud computing: A survey. International Journal of Computer Applications, 86(16):18-21, January 2014.

[25] Gulshan Soni and Mala Kalra. A comparative study of live virtual machine migration techniques in cloud. International Journal of Computer Applications, 84(14):19-25, December 2013.

[26] Anja Strunk. Costs of virtual machine live migration: A survey. In Services (SERVICES), 2012 IEEE Eighth World Congress on, pages 323-329. IEEE, 2012.

[27] Matheus Torquato, Jean Araujo, and Paulo Maciel. Estudo experimental de envelhecimento de software em nuvens kvm/opennebula: Live migration como mecanismo de suporte 
ao rejuvenescimento de software. In XIII Workshop de Computao em Clouds e Aplicaes (WCGA 2015), em conjunto com o 33 Simpsio Brasileiro de Redes de Computadores e Sistemas Distribudos (SBRC 2015), 2015.

[28] William Voorsluys, James Broberg, Srikumar Venugopal, and Rajkumar Buyya. Cost of virtual machine live migration in clouds: A performance evaluation. In Cloud Computing, pages 254-265. Springer, 2009.

[29] A.J. Younge, R. Henschel, J.T. Brown, G. von Laszewski, J. Qiu, and G.C. Fox. Analysis of virtualization technologies for high performance computing environments. In Cloud Computing (CLOUD), 2011 IEEE International Conference on, pages 9-16, July 2011.

[30] Ming Zhao and Renato J. Figueiredo. Experimental study of virtual machine migration in support of reservation of cluster resources. In Proceedings of the 2Nd International Workshop on Virtualization Technology in Distributed Computing, VTDC '07, pages 5:1-5:8, New York, NY, USA, 2007. ACM. 